\title{
Pulsar Wind Nebulae in EGRET Error Boxes
}

\author{
Mallory S.E. Roberts (roberts@physics.mcgill.ca) \\ McGill University / Eureka Scientific
}

Crystal L. Brogan, Bryan M. Gaensler and Jason W.T. Hessels

Institute for Astronomy, Harvard-Smithsonian CfA, and McGill U.

C.-Y. Ng and Roger W. Romani

Stanford University

June 2, 2004

\begin{abstract}
A remarkable number of pulsar wind nebulae (PWN) are coincident with EGRET $\gamma$-ray sources. X-ray and radio imaging studies of unidentified EGRET sources have resulted in the discovery of at least 6 new pulsar wind nebulae (PWN). Stationary PWN (SPWN) appear to be associated with steady EGRET sources with hard spectra, typical for $\gamma$-ray pulsars. Their toroidal morphologies can help determine the geometry of the pulsar which is useful for constraining models of pulsed $\gamma$-ray emission. Rapidly moving PWN (RPWN) with more cometary morphologies seem to be associated with variable EGRET sources in regions where the ambient medium is dense compared to what is typical for the ISM.
\end{abstract}

Keywords: PWN, EGRET, PSR J2021+3651, GeV J1809-2327, Rabbit, GammaRay

\section{Coincidence of PWN with EGRET Sources}

A pulsar wind nebula (PWN) is a X-ray, radio, and/or $\mathrm{H} \alpha$ nebula caused by a relativistic particle wind from an energetic pulsar (see Kaspi et al. (2004) and Gaensler (2004) for recent reviews). Of the $\sim 50$ known PWN, a large fraction are coincident with EGRET sources. If we consider only the $\sim 30$ sources with significant emission above $1 \mathrm{GeV}$ (listed in the catalog of Lamb and Macomb (1997)) which are probably associated with our Galaxy, nearly half the error ellipses contain a known PWN, and several of the others have not yet been searched carefully for X-ray nebulae. In Table I, we list all the known PWN coincident with EGRET sources (for references to these sources, see http://www.physics.mcgill.ca/ pulsar/pwncat.html). We also list the morphological type ( $\mathrm{S}$ or $\mathrm{R}$, see below) if it can be determined, the pulsar spin-down energy $\dot{E}$ if pulsations have been detected, and the variability index $V_{12}$ of the EGRET source as defined and determined by Nolan et al. (2003). $10^{-V_{12}}$ is the integrated liklihood that the variability measure $\tau \equiv \sigma_{F} /<F>\leq 0.12$, or the $>100 \mathrm{MeV}$ flux is consistent with being constant. In other words, $V_{12}>1$ indicates a $>90 \%$ confidence level that the $\gamma$-ray source is variable. No value indicates there is no evidence for variability.

(C) 2018 Kluwer Academic Publishers. Printed in the Netherlands.

mroberts.tex; 12/11/2018; 3:27; p.1 
Table I. Pulsar Wind Nebulae Coincident with EGRET Sources

\begin{tabular}{|c|c|c|c|c|c|}
\hline Name & 3EG Name & GeV Name & Type & $\log \dot{E}$ & $V_{12}$ \\
\hline CTA 1 & 3EG J0010+7309 & GeV J0008+7304 & $?$ & - & 0.40 \\
\hline Crab & $3 \mathrm{EG}$ J0534+2200 & GeV J0534+2159 & $\mathrm{S}$ & 38.7 & - \\
\hline Geminga & 3EG J0633+1751 & GeV J0634+1746 & $\mathrm{R}$ & 34.5 & 0.16 \\
\hline Vela & 3EG J0834-4511 & GeV J0835-4512 & $\mathrm{S}$ & 36.8 & 0.61 \\
\hline MSH 11-62 & 3EG J1102-6103 & - & $\mathrm{R}$ & - & - \\
\hline PSR J1016-5857 & 3EG J1013-5915 & - & $?$ & 36.4 & 0.18 \\
\hline PSR B1046-58 & 3EG J1048-5840 & GeV J1047-5840 & $?$ & 36.3 & - \\
\hline PSR J1420-6048 & 3EG J1420-6038 & GeV J1417-6100 & $?$ & 37.0 & 1.59 \\
\hline Rabbit & 3EG J1420-6038 & GeV J1417-6100 & $\mathrm{R}$ & - & 1.59 \\
\hline PSR B1706-44 & 3EG J1710-4439 & GeV J1709-4430 & $\mathrm{S}$ & 36.5 & - \\
\hline G359.89-0.08 & 3EG J1746-2851 & GeV J1746-2854 & $\mathrm{R}$ & - & 2.35 \\
\hline G7.4-2.0 & 3EG J1809-2328 & GeV J1809-2327 & $\mathrm{R}$ & - & 3.93 \\
\hline G18.5-0.4 & 3EG J1826-1302 & GeV J1825-1310 & $\mathrm{R}$ & - & 3.22 \\
\hline PSR B1853+01 & 3EG J1856+0114 & GeV J1856+0115 & $\mathrm{R}$ & 35.6 & 1.57 \\
\hline $3 \mathrm{C} 396$ & 3EG J1903+0550 & - & $?$ & - & 0.42 \\
\hline CTB 87 & $3 \mathrm{EG}$ J2016+3657 & - & $?$ & - & 0.63 \\
\hline PSR J2021+3651 & $3 \mathrm{EG}$ J2021+3716 & GeV J2020+3658 & $\mathrm{S}$ & 36.5 & 0.71 \\
\hline PSR J2229+6114 & $3 \mathrm{EG}$ J2227+6122 & $\mathrm{GeV} \mathrm{J} 2227+6101$ & $\mathrm{~S}$ & 37.4 & 0.21 \\
\hline
\end{tabular}

At least six of these PWN were discovered through X-ray searches of the EGRET error box, indicating that whatever the physical association, $\gamma$-ray sources in the Galactic plane can serve as guides to the location of energetic pulsars that otherwise might be difficult to find.

\section{Pulsar Geometry From Stationary Pulsar Wind Nebulae}

The environment of a young pulsar is the expanding ejecta of its progenitor star. This allows for fast expansion of the young PWN relative to the kick velocity imparted to the pulsar. Therefore, the motion of the pulsar does not greatly affect the structure of the PWN. The underlying symmettries of the pulsar's spinning magnetic field are then reflected in the structure of the X-ray emitting PWN. We refer to these as stationary PWN (SPWN). SPWN tend to have some sort of equatorial toroidal structure and polar jets. The apparent ellipticity and brightness variations of these toroidal structures can be used to infer the orientation of the pulsar's spin axis relative to the observer. In addition, the magnetic inclination angle may relate to the thickness of the torus in the case of single or concentric torii such as the Crab, or the separation between two parallel torii such as is apparently the case for the Vela PWN. For details in the methodology used to model observed SPWN torii, see Ng and Romani (2004). 
This geometrical information is crucial for interpreting $\gamma$-ray pulse profiles and hence understanding the particle acceleration mechanisms in pulsar magnetospheres. It is therefore important to infer the geometry of many torii around $\gamma$-ray emitting pulsars. Unfortunately, in only a handful of cases is the PWN structure clear enough for unambiguous interpretation of the geometry, even with the high resolution of the Chandra X-ray observatory. Only three of the known $\gamma$-ray pulsars (Crab, Vela, and PSR B1706-44) have torii amenable to fitting. It is therefore important to expand the number of potential $\gamma$-ray emitting pulsars with torii. X-ray imaging of unidentified EGRET source error boxes has resulted in the detection of two energetic young pulsars with well structured nebulae: PSR J2229+6114 (Halpern et al., 2001) and PSR J2021+3651 (Hessels et al., 2004).

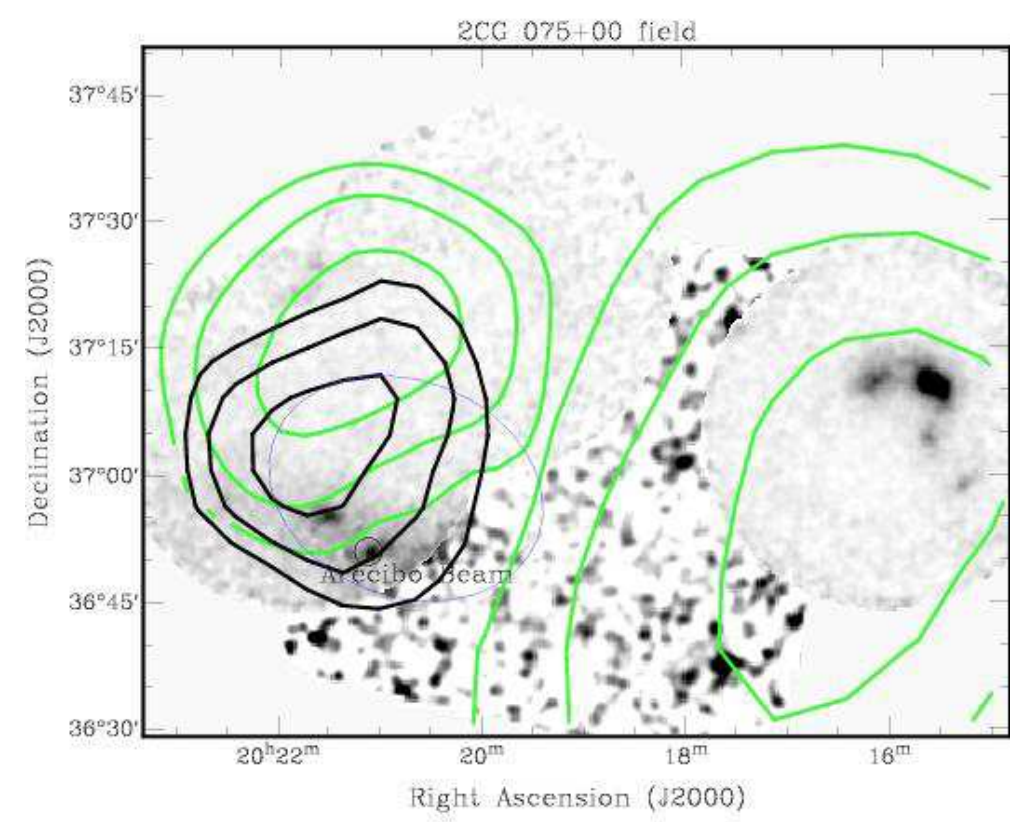

Figure 1. X-ray image of the region of the COS B source 2CG $075+00$ containing two 3rd EGRET Catalog sources (light green contours), one GeV Catalog source (ellipse) and the refit $\mathrm{GeV}$ contours. The Arecibo beam pointing is shown which was used to discover PSR J2021+3651.

The methodology used to discover PSR J2021+3651 is illustrative of how to discover and measure usable torii. The $\gamma$-ray source is in the crowded Cygnus region, which contains two highly significant GeV catalog sources which are not listed in the 3rd EGRET Catalog. A refitting of the region using only photons above $1 \mathrm{GeV}$ but including all known EGRET sources was performed in order to obtain the best position (see Fig. 1). A low resolution, wide field, hard X-ray observation was made 
using the $A S C A$ GIS detectors, discovering an apparent point source at the $\sim 1^{\prime}$ resolution of $A S C A$ (Roberts et al., 2001). This allowed the $3^{\prime}$ beam of the Arecibo telescope to perform a very deep search of the source to discover the $\sim 0.1 \mathrm{mJy}$ pulsar with spin properties virtually identical to PSR B1706-44 (Roberts et al., 2002). A $20 \mathrm{ks}$ Chandra $A C I S$ image of the X-ray source revealed a compact nebula with a morphology highly suggestive of a double torus with polar jets. However, the scarcity of counts in the image leaves some ambiguity as to whether or not it is a single or double torus, and whether the jets are real (Fig. 2).
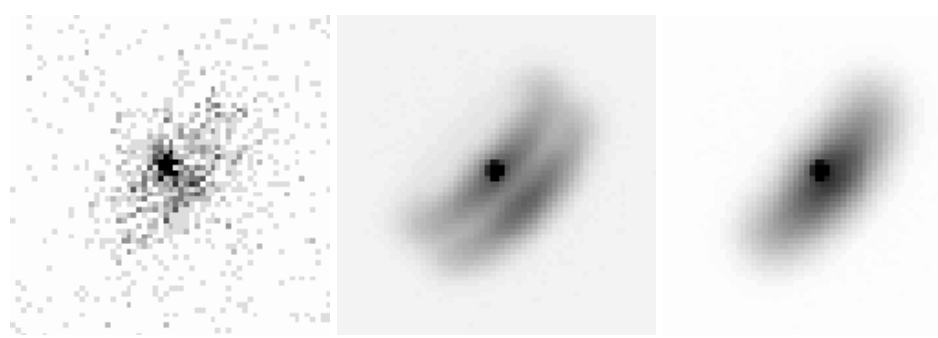

Figure 2. Chandra image of PSR J2021+3651 with best fit double and single torus models (Hessels et al. 2004).

If the double torus interpretation is made, then the separation of the two torii can be assumed to be an indication of the magnetic inclination angle. This can be sanity checked by looking at the intrinsic radio pulse width, which may indicate whether the pulse is predominantly a core or conal component, and by measuring the radio polarization sweep and fitting it to a Radhakrishnan and Cooke (1969) model.

Unfortunately, the above 5 sources are the only known pulsars associated with EGRET sources whose nebulae are large enough and bright enough to be useful for fitting. However, there are several more PWN with toroidal morphologies which either do not yet have detected pulsations or are not yet associated with a $\gamma$-ray source. Even with $G L A S T$, it is likely that only $\sim 10 \gamma$-ray pulsars will be found whose geometry can be well constrained by their nebulae with Chandra imaging. We can only hope that will be enough to determine the location in the magnetosphere where $\gamma$-rays are emitted.

\section{Rapidly Moving Pulsar Wind Nebulae Associated With Variable EGRET Sources}

The SPWN discussed above tend to be associated with hard spectrum $(\Gamma \lesssim 2)$, steady $\left(V_{12}<1\right)$ EGRET sources. Such sources tend to be nearly $100 \%$ pulsed in $100-10000 \mathrm{MeV} \gamma$-rays, and be the youngest 
and most energetic pulsars. After a few thousand years, the reverse shock of the supernova blast wave reaches the PWN, after which the environment is no longer the expanding ejecta causing the PWN expansion to slow dramatically and become subsonic. If the pulsar has a significant space velocity, it will start to catch up to the forward edge of the PWN, drastically affecting the morphology as ram-pressure becomes more important. If the space velocity exceeds the local sound speed, then the PWN will be confined in the forward direction by a bowshock. These cometary shaped nebulae we refer to as rapidly-moving or ram-pressure confined PWN (RPWN). In X-rays, they generally have a narrow outflow trailing the pulsar, with the pulsar itself typically having a spin-down energy such that $\log \dot{E} \sim 35.5-36.5 \mathrm{erg} / \mathrm{s}$, somewhat less than the typical SPWN. This is expected given their on average greater age.

Perhaps the best example of a RPWN is the Mouse nebula near the Galactic center. The X-ray nebula of the Mouse is significantly brighter than other RPWN, and consequently shows significantly more structure (Fig. 3). A "tongue" of brighter emission is surrounded by a fainter halo, which is contained within the bright radio "head" of the Mouse. A fainter radio body extends back from the head, which eventually narrows into an extremely long tail (Gaensler et al., 2003). Hydrodynamic models suggest the tongue is associated with the wind termination shock. However, much of the structure, especially downstream of the head, is currently not understood. The generally short Chandra observations of other RPWN contain very few counts, and only the structure corresponding to the Mouse's "tongue" is clearly delineated.

Remarkably, several RPWN have been discovered in the error boxes of EGRET sources which are apparently variable. The source with the highest $V_{12}$ value in the Galactic plane without a potential Blazar identification is GeV J1809-2327. An X-ray nebulae was discovered in its error box with $A S C A$ (Roberts et al., 2001) which subsequent Chandra and VLA imaging showed to be a RPWN (Braje et al., 2002). An XMM-Newton EPIC-PN observation, which was unfortunately taken in small window mode, suggests a fainter X-ray halo surrounding the $\mathrm{X}$-ray trail seen in the short Chandra exposure, strongly reminiscent of the Mouse (Fig. 4). The X-ray emission seen in the EPIC-PN image is coincident with the front edge of the radio PWN, itself reminiscent of the head of the Mouse, with the suggestion of a fainter, extended body to the Northwest. The radio and X-ray emission seems to extend back towards a possible X-ray SNR, G7.4-1.4, discovered by ROSAT. Widefield $90 \mathrm{~cm}$ imaging is underway to determine if there is a corresponding radio SNR. The PWN appears to be embedded in the Lynds 227 dark 


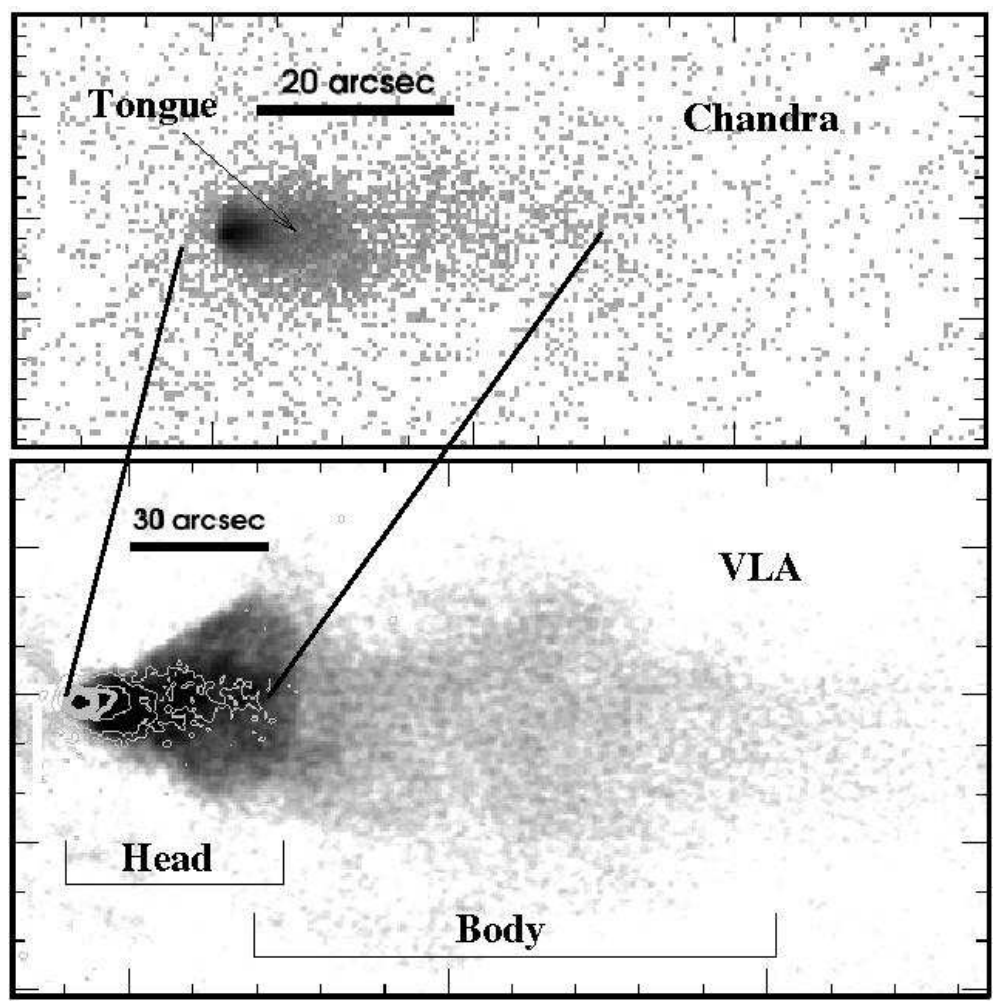

Figure 3. Chandra X-ray and VLA radio images of the Mouse nebula showing the various structures (Gaensler et al. 2003).

Nebula, and CO imaging suggests a physical connection between the PWN and the molecular cloud (Oka et al., 1999).

The region near Galactic longitude $l=18^{\circ}$ contains several EGRET sources, one or more of which are probably associated with the well known unidentified 1-30 MeV Comptel source (see Collmar et al., these proceedings). Wide-field radio imaging (Brogan et al. in preparation) of the region shows several SNR and the Sharpless 53 cluster of HII regions. One of the EGRET sources, GeV J1825-1310, has the second highest $V_{12}$ value of Galactic plane sources. $A S C A$ imaging of this source followed by a short Chandra image again revealed a faint RPWN in a stellar cluster (Romani et al. in preparation). There is some hint of a larger radio nebula in the $90 \mathrm{~cm}$ image.

Two other sources with $V_{12}>1.3$ (95\% confidence of variability) contain apparent RPWN. One is the PWN around PSR B1853+01 in the X-ray composite SNR W44 (Petre et al., 2002). The other is the source containing the Kookaburra radio complex, the wings of which contain the PWN around PSR J1420-6048 and the Rabbit PWN (Roberts 

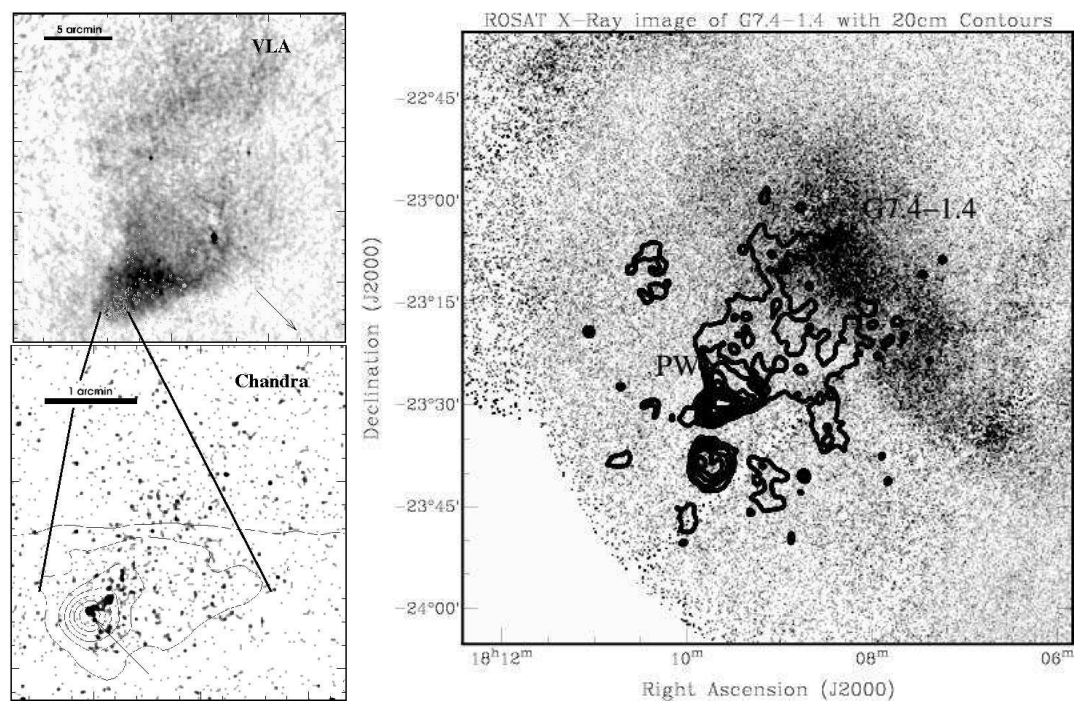

Figure 4. Left: VLA and Chandra images of the PWN in GeV J1809-2327. The contours on the Chandra image are from the EPIC-PN, which are cut off due to the small window used. Right: ROSAT image of the SNR G7.4-1.4, with contours from the VLA image of the PWN.

et al., 1999). Chandra and XMM-Newton imaging of the Rabbit nebula (Ng et al., Roberts et al. in preparation) show the X-ray emission going from a point source in the front paw back towards the body and the southern wing of the Kookaburra. This strongly suggests the Rabbit is the head of a RPWN, with the wing being the fainter body. In addition to these sources, there is a probable RPWN, G369.89-0.08 (Lu et al., 2003), within the error box of the variable source near the Galactic center, which the Mouse is very near as well.

Overall, around half of the Galactic plane sources with good evidence of variability and no candidate Blazar counterpart seem to contain RPWN. All of them also seem to be in regions containing molecular clouds or other evidence of relatively high densities in the ambient medium. This suggests the $\gamma$-ray emission may be associated with the pulsar passing through local density enhancements.

\section{Conclusions}

There is an intimate relationship between the generation of PWN and of $\gamma$-ray emission. This is not surprising since both indicate particles being accelerated to very high energies in the pulsar magnetosphere. Because of this, $\gamma$-ray sources in the Galaxy tend to pick out PWN that otherwise might be hard to find in the crowded Galactic plane. SPWN with clear toroidal morphologies are key to determining pulsar geome- 
tries, but require deep Chandra imaging. No other X-ray telescope now operating or currently planned has the necessary resolution. RPWN appear to be associated with variable EGRET sources, which AGILE and GLAST should easily confirm. However, there is no real theory developed yet as to how they might be generating $\gamma$-ray emission, and much deeper Chandra X-ray imaging is required to determine their true structure.

\section{References}

Braje, T. M., R. W. Romani, M. S. E. Roberts, and N. Kawai: 2002, 'Chandra Imaging of the Gamma-Ray Source GeV J1809-2327'. ApJ 565, L91-L95.

Gaensler, B. M.: 2004, 'Shocks and Wind Bubbles Around Energetic Pulsars'. In: IAU Symposium. pp. 151-+.

Gaensler, B. M., E. van der Swaluw, F. Camilo, V. M. Kaspi, F. K. Baganoff, F. Yusef-Zadeh, and R. N. Manchester: 2003, 'The Mouse That Soared: High Resolution X-ray Imaging of the Pulsar-Powered Bow Shock G359.23-0.82'. ArXiv Astrophysics e-prints.

Halpern, J. P., F. Camilo, E. V. Gotthelf, D. J. Helfand, M. Kramer, A. G. Lyne, K. M. Leighly, and M. Eracleous: 2001, 'PSR J2229+6114: Discovery of an Energetic Young Pulsar in the Error Box of the EGRET Source 3EG J2227+6122'. ApJ 552, L125-L128.

Hessels, J. W. T., M. S. E. Roberts, S. M. Ransom, V. M. Kaspi, R. W. Romani, C. . Ng, P. C. C. Freire, and B. M. Gaensler: 2004, 'Observations of PSR J2021+3651 and its X-ray Pulsar Wind Nebula G75.2+0.1'. ApJ 612, 389-397.

Kaspi, V. M., M. S. E. Roberts, and A. K. Harding: 2004, 'Isolated Neutron Stars'. ArXiv Astrophysics e-prints.

Lamb, R. C. and D. J. Macomb: 1997, 'Point Sources of GeV Gamma Rays'. ApJ 488, 872 .

Lu, F. J., Q. D. Wang, and C. C. Lang: 2003, 'The Chandra Detection of Galactic Center X-Ray Features G359.89-0.08 and G359.54+0.18'. AJ 126, 319-326.

Ng, C.-Y. and R. W. Romani: 2004, 'Fitting Pulsar Wind Tori'. ApJ 601, 479-484.

Nolan, P. L., W. F. Tompkins, I. A. Grenier, and P. F. Michelson: 2003, 'Variability of EGRET Gamma-Ray Sources'. ApJ 597, 615-627.

Oka, T., N. Kawai, T. Naito, T. Horiuchi, M. Namiki, Y. Saito, R. W. Romani, and T. Kifune: 1999, 'A Dark Cloud Associated with an Unidentified EGRET Source'. ApJ 526, 764-771.

Petre, R., K. D. Kuntz, and R. L. Shelton: 2002, 'The X-Ray Structure and Spectrum of the Pulsar Wind Nebula Surrounding PSR B1853+01 in W44'. ApJ 579, 404-410.

Radhakrishnan, V. and D. J. Cooke: 1969, 'Magnetic poles and the polarization structure of pulsar radiation'. Astrophys. Lett. 3, 225-229.

Roberts, M. S. E., J. W. T. Hessels, S. M. Ransom, V. M. Kaspi, P. C. C. Freire, F. Crawford, and D. R. Lorimer: 2002, 'PSR J2021+3651: A Young Radio Pulsar Coincident with an Unidentified EGRET $\gamma$-Ray Source'. ApJ 577, L19-L22.

Roberts, M. S. E., R. W. Romani, S. Johnston, and A. J. Green: 1999, 'The "Rabbit": A Potential Radio Counterpart of GeV J1417-6100'. ApJ 515, 712-720.

Roberts, M. S. E., R. W. Romani, and N. Kawai: 2001, 'The ASCA Catalog of Potential X-Ray Counterparts of GeV Sources'. ApJS 133, 451-465. 